\title{
NUMERICAL MODEL APPLICATION TO PREDICT THE SOUND QUALITY OF AN INSTRUMENT
}

\author{
Ray, T.; Kaljun, J. \& Dolsak, B. \\ University of Maribor, Faculty of Mechanical Engineering, Smetanova 17, SI-2000 Maribor, Slovenia \\ E-Mail: tonyray@t-2.si, jasmin.kaljun@um.si,bojan.dolsak@um.si
}

\begin{abstract}
It is well known that in an acoustic guitar, the hollow wooden body of the guitar, which forms the resonating chamber, plays an important role in the sound produced. In an electric guitar, the influence of the solid guitar body is not so crucial, yet some influence of the vibroacoustic material properties is expected but has not been scientifically confirmed so far. The aim of the research presented in this paper was to confirm the hypothesis and to investigate the influence of the material used for the solid body of an electric guitar on the final sound image. An experimental numerical model of the electric guitar was created to show how designers can be assisted with a tool that allows them to plan the characteristics of the sound to be produced at the design stage, when the material and shape for the body of the guitar have been determined. In order to develop a relevant numerical model of the electric guitar, we built a test bench to validate the numerical model. The results of the numerical simulation of the decay of the string vibrations as a function of the body material used agree well with the results of the experimental measurements, confirming the adequacy of the numerical model presented.

(Received in August 2021, accepted in November 2021. This paper was with the authors 1 month for 1 revision.)
\end{abstract}

Key Words: Numerical Analysis, Simulation, Electric Guitar, Wooden Solid Body, Vibroacoustic Properties

\section{INTRODUCTION}

\subsection{Electric solid body guitar}

Electric solid body guitar is a musical instrument that produces sound through the principle of mechanical vibration of the strings. These vibrations are transmitted to the pickup that sends the signal to the amplifier, which emits the sound through the speakers [1]. For the good sound of the instrument, a good choice of pickups and strings alone is not enough. The right choice of materials for the body and neck of the guitar and of course the shape of these two parts is also important, as the vibroacoustic properties of the materials have a decisive influence on the final image of the sound of the musical instrument.

\subsection{Material impact}

The function and effect of wood in electric instruments is still not fully understood. Most of the controversy relates to the colour of the sound or to the sound itself. Opinions are very much divided on the subject. Wood is a natural composite material. As a component of musical instruments, it plays an important role in the formation of sound and contributes to their appeal and also to their cultural identity.

There are various kinds of wood used in the manufacture of musical instruments. In this field, it is not only the acoustic aspect that is important, but also the mechanical behaviour, physical stability, visual aesthetics and tactile properties that are very important. Due to the very different sound properties of the individual types of wood, different instruments can be made from different types of wood.

Noguchi et al. state that the knowledge of wood structure is very important to identify the materials used for the construction of musical instruments. The physical, mechanical and 
chemical properties of wood are strongly influenced by the arrangement and quantity of the constitutive components (cellulose, lignin, hemicelluloses) and the wood structure [2].

Interesting is the study of Voichita Bucur, which describes the mechanical properties of wood (elastic properties, Young's modulus, Poisson's ratio, stiffness modulus) and the vibration properties of wood [3].

Wood fibres having a tubular structure are able to accumulate a larger volume of air in the fibres than in the cell walls. This fact has a significant impact on the speed of sound waves in the acoustic properties of each type of wood, which is very important when wood is used for making musical instruments, Smardzewski et al. write in their study [4].

\subsection{Sound image and subjective impression}

To determine the influence of the material on the sound of the electric guitar, we first conducted a survey on the World Wide Web. We collected a large amount of information suggesting that the material of the guitar body has an influence on the sound produced [5], but this was not scientifically confirmed (Fig. 1). Therefore, in our initial investigation, we took measurements of the sound of two sample guitars that differed only in body material, one made of walnut and the other of ash.

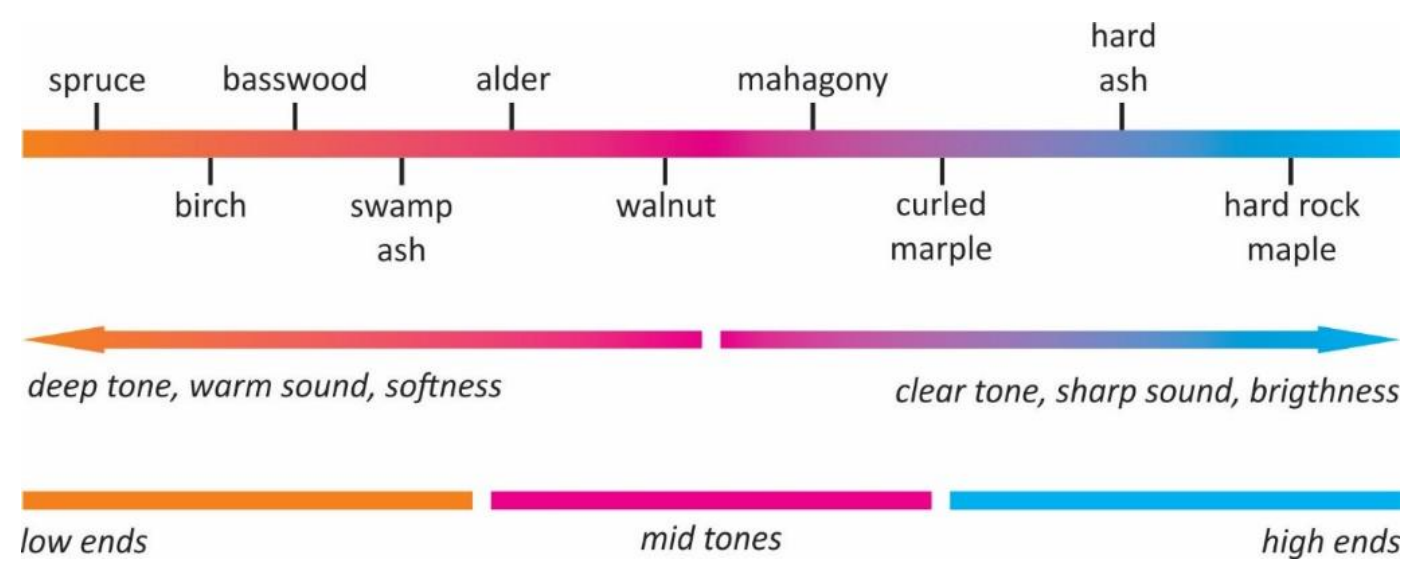

Figure 1: Subjective impression of sound according to guitar body material (wood).

\subsection{The process of designing the body of a guitar using numerical methods}

When constructing an electric guitar with a solid body, the acoustic properties of the guitar (the sound produced) depend on both the shape of the guitar body and the material with its characteristic physical and technical properties.

The process of electric guitar design driven by acoustic measurements (Fig. 2) begins with the definition or characterization of the material from which the body of the electric guitar is going to be made [5]. It is important to first establish or define a measurement protocol that will be the starting point for further consideration of the design and construction recommendations of the design methodology [6].

A key element in the presented process of designing an electric guitar is the implementation of numerical methods to simulate or evaluate the sound of the resulting instrument in a virtual environment. Practically all numerical simulations are based on the definition of three basic elements: geometry, material model and boundary conditions, on the basis of which the desired data are calculated using an appropriate numerical method or various optimization processes are performed $[7,8]$. Moreover, based on the results of numerical analysis, new additive manufacturing processes can be planned [9] to produce bodies with desired properties. 


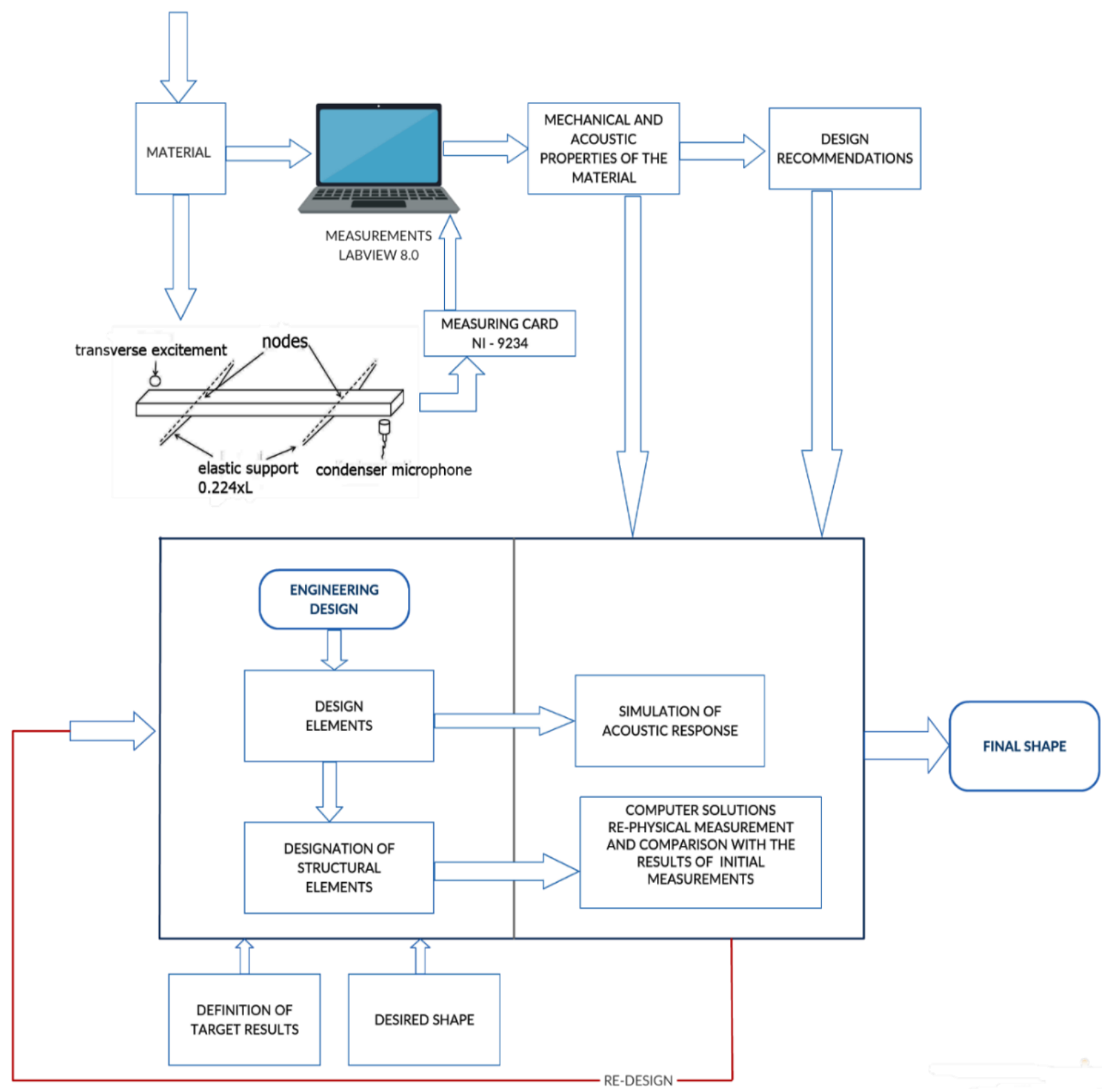

Figure 2: Electric guitar design driven by acoustic measurements.

In this paper, we present a process of developing and validating a numerical model of the string vibration of an electric guitar that incorporates the influence of the guitar body material. The development of the numerical model started with laboratory measurements of the damping of the string vibration on different guitar configurations. Based on the results of the measurements, the geometry of the numerical model was defined. This was followed by the definition of the material properties of the individual elements and the determination of the numerical simulation procedure.

\section{METHODS AND MATERIALS}

In order to establish a protocol for the design of an electric guitar, we divided our research into three basic parts: the design of the body of the electric guitar [1]; the definition of the mechanical and acoustic properties of the material, in our case natural wood [6]; and the numerical simulation of the string vibration decay affected by the body material used, which is the main topic of this paper. 
In order to develop a suitable and also practically applicable numerical model of the electric guitar, we built a test bench to validate the numerical model. However, before defining the numerical model, we need to deal with the structure of the full-body electric guitar. Fig. 3 shows a typical guitar configuration with the main elements.

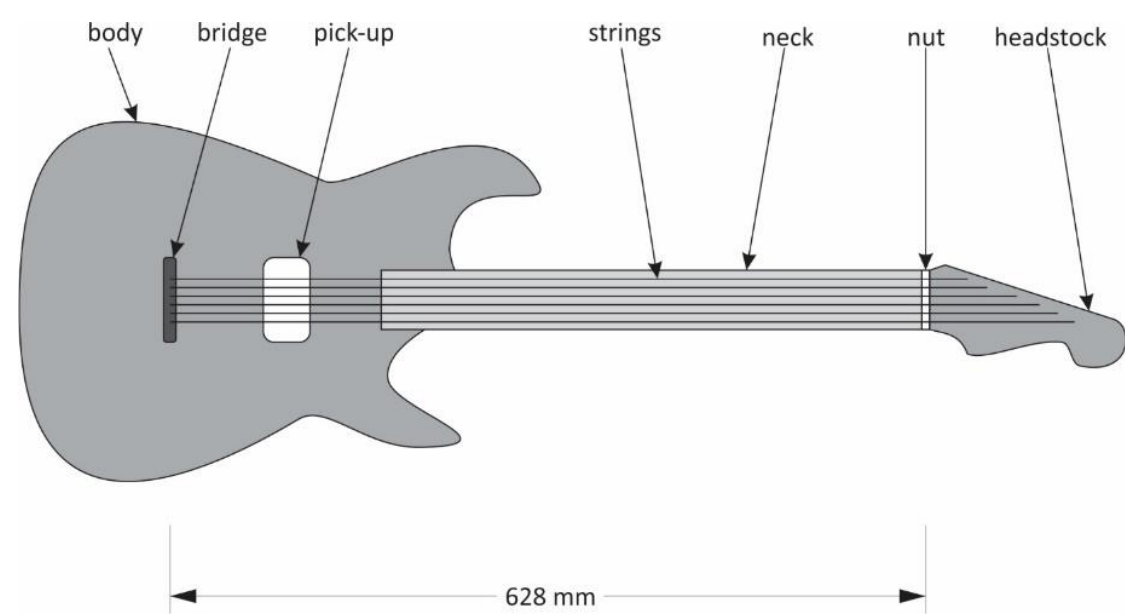

Figure 3: Typical elements of solid body electric guitar.

Since we only want to analyse the influence of the guitar body on the final sound, we idealised the guitar by keeping only the relevant elements, i.e. the guitar body and the string. To verify the idealisation, two sets of measurements were performed on the test bench, with the configurations shown in Fig. 4. In the first case (Fig. 4 a), we kept the guitar body and bridge on the body, while removing the neck and headstock and replacing them with a fastener over which we stretched the string. In this case, the pickup was placed in three locations: in the body of the guitar (standard guitar mount); at the point of maximum string amplitude in the first vibration mode; and above the body, over the standard pickup mount. In the second example (Fig. 4 b), the guitar body was fitted with a conventional neck and headstock, and the pickup was placed in the guitar body (standard guitar mount).
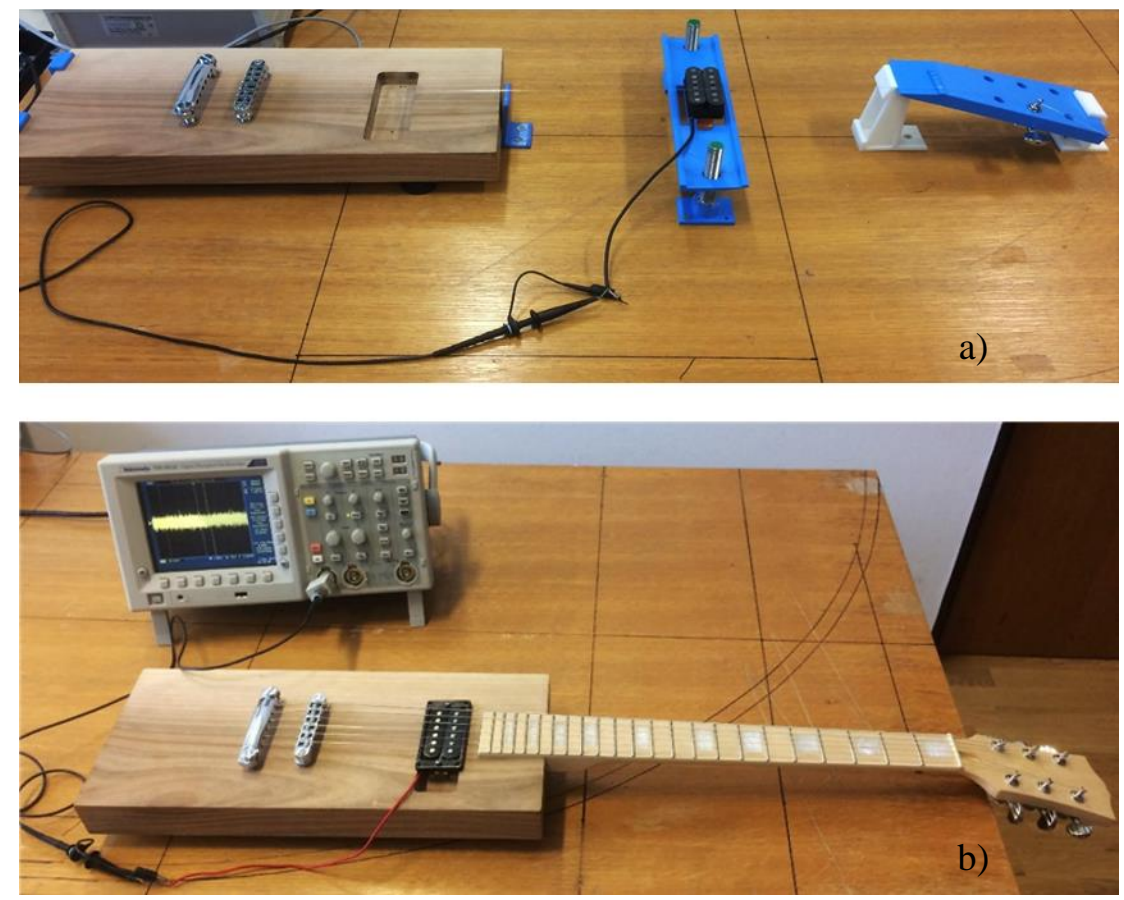

Figure 4: Configurations for the laboratory measurements. 
The string (a normal steel string) was tuned to the tone G, i.e. to a fundamental oscillation frequency of $196 \mathrm{~Hz}$. The microclimatic conditions in the laboratory were matched to those of the measurements of the vibro-mechanical properties of the wood $\left(T=20^{\circ} \mathrm{C}, \varphi=50 \%\right)$. The wood of the guitar body was naturally dried and conditioned.

Ten consecutive measurements of the induced electrical voltage in a magnetic pickup (DiMarzio DP103 PAF $36^{\text {th }}$ Anniversary) were made, with a time interval of $5 \mathrm{~s}$ and a step of $1 \mathrm{~ms}$. The string was plucked with a horizontal (parallel to the table surface) force of $5 \mathrm{~N}$. A Tektronix TDS 3012C Digital Phosphor oscilloscope was used for the measurements.

The electromagnetics of an electric guitar effectively converts the mechanical energy present in a vibrating guitar string into an oscillating voltage (as the guitar strings vibrate over the guitar's magnetic pickup, the time-varying magnetic flux through the metal strings creates an oscillating voltage inside the coil of the magnetic pickup). It turns out that electromagnetic theory predicts, at least within ideal damping effects, that the displacements of the guitar string, neglecting some phase change, are directly proportional to the oscillating voltages induced by these vibrations [10].

The results obtained were processed and normalised to ensure the comparability of the individual measurements. The idealised data of the oscillation voltage over the time interval were then compared. Fig. 5 shows the results of the measurements for both experimental configurations.
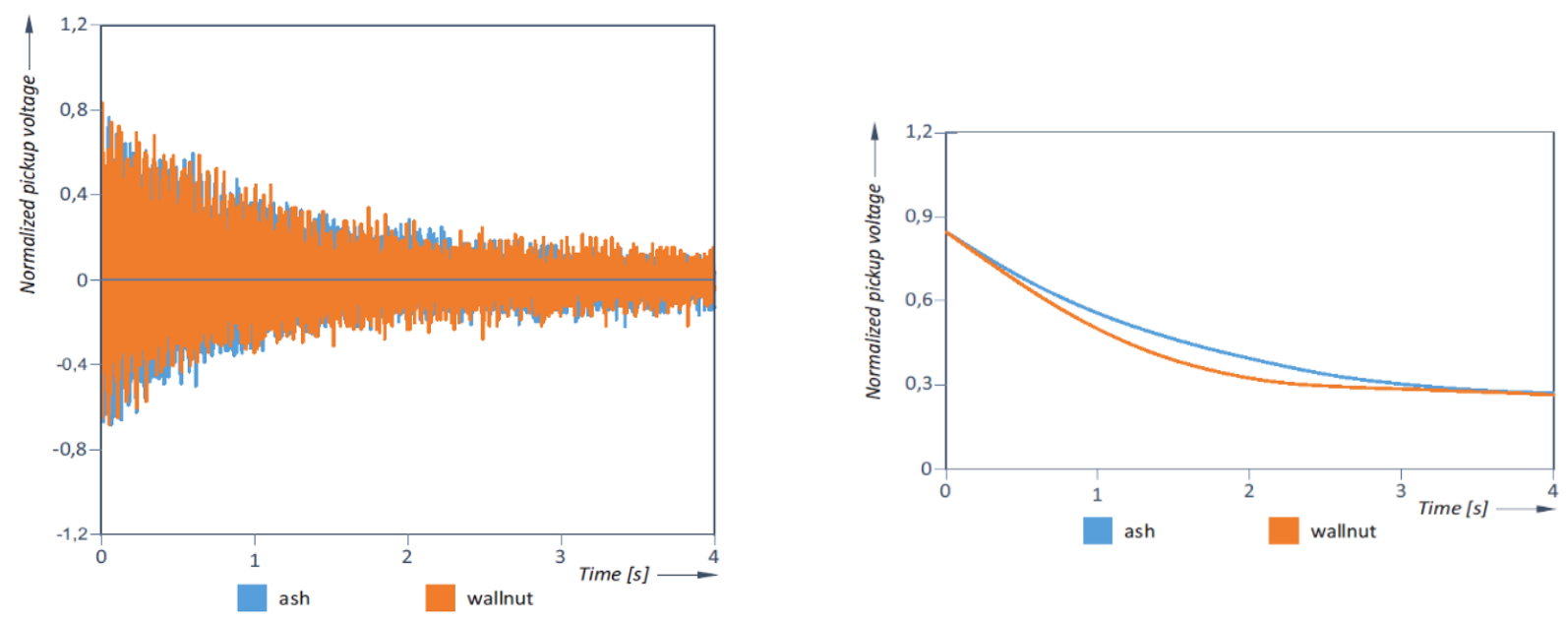

a)
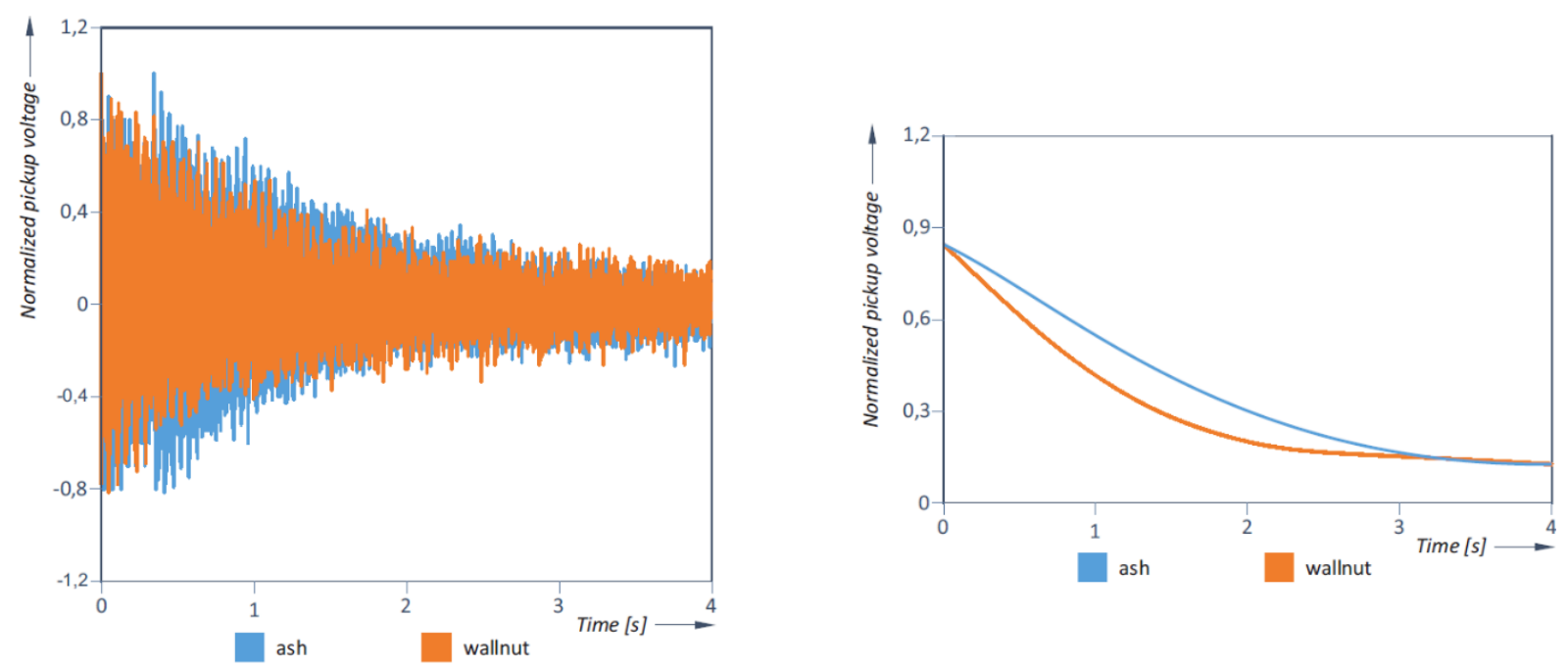

b)

Figure 5: The curves of the induced voltage and the comparison of the decay curves. 
As can be seen in Fig. 5, the difference in produced sound is clearly visible at graph envelopes. In the case of the walnut body of the guitar, a faster damping of the vibrations is observed. It can be concluded that the neck and headstock connected to the guitar body (Fig. $5 \mathrm{~b})$ do not have a significant effect on the sound of the guitar. When the same neck and headstock are used, the acoustic image depends on the body.

This finding was subsequently taken into account in the construction of a numerical model of an electric guitar with a solid body, the purpose of which is a virtual analysis of the damping of the string vibrations and thus the sound image produced.

\section{NUMERICAL MODEL}

The use of numerical analysis goes beyond classical engineering problems. Numerical analysis is also increasingly used in areas where boundary conditions are not uniquely determined $[11,12]$ and in complex optimization processes [13]. Also in the presented case, the correct definition of boundary conditions and material parameters as well as the connexions between the individual geometric elements of the numerical model are of crucial importance.

Using ANSYS 2020 R2, we developed a numerical model of the body-string system of an electric guitar to numerically simulate laboratory measurements. A transient structural analysis with Mechanical APDL solver was chosen as the initial analysis system.

The geometry and material data of the model (Fig. 6) correspond to the real model as in the laboratory measurements. The G-string was defined and included in the numerical model. The length of the loaded string between the clamps was $628 \mathrm{~mm}$.

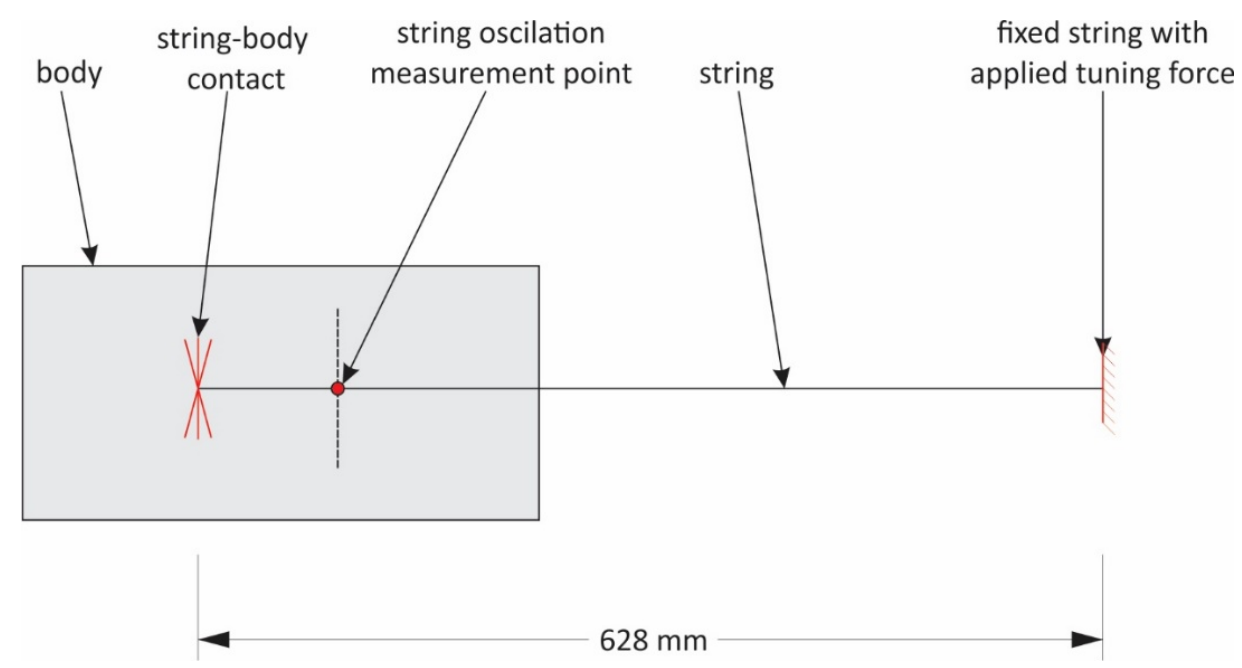

Figure 6: Schematic representation of the model for transient structural analysis.

The string was connected to the body by a "fixed connection" with "remote attachment" at the holes for bridge screws (Fig. 7). We defined a deformable connection that ensures the transmission of mechanical (vibration) reactions between the body and the string. On the other side, at the location of the nut, we applied the string tension force in the longitudinal direction of the string (Fig. 6). The body supports were placed so that they matched the supports in the laboratory measurements. The height of the string above the guitar body was $14 \mathrm{~mm}$. A force of $5 \mathrm{~N}$ (Table I) was applied to the string between the two holes for the pickups. The string tension depended on the diameter and material of the string. The whole testing procedure was divided into four steps, which lasted $4 \mathrm{~s}$ in total. Data were collected at time intervals of $0.001 \mathrm{~s}$. The data and the final graphs were processed using FlexPro 7 software. The analysis considered the damping of ash and walnut wood to determine the influence of the body on the vibration of the strings. 


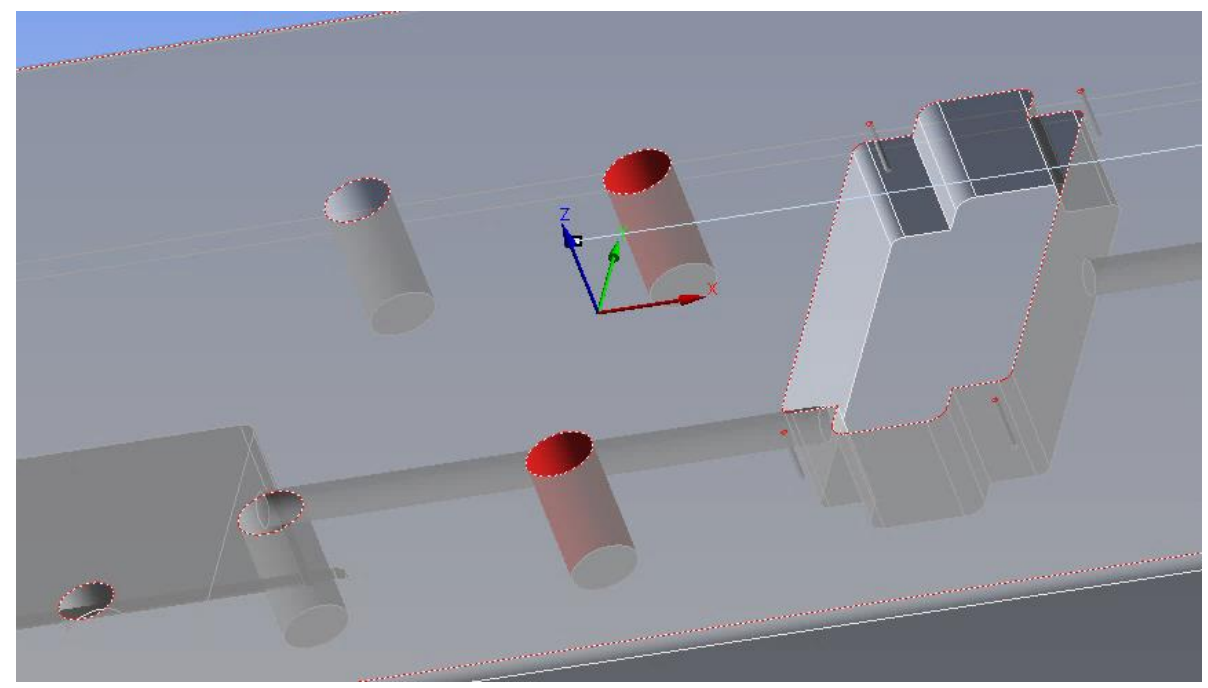

Figure 7: Virtual connection of string and body.

Special attention was paid to a correct definition of the material model. According to the transient structural analysis simulation, we defined the material model based on the standard mechanical properties of wood [5] determined in the laboratory and specified a suitable damping model. We chose the Rayleigh damping model, which assumes that the damping matrix is proportional to the mass and stiffness matrix, Eq. (1) [14]:

$C$ - damping matrix,

$$
C=\alpha M+\beta K
$$

$M$ - mass matrix,

$\alpha$ - Rayleigh damping coefficient (mass),

$\beta$ - Rayleigh damping coefficient (stiffness).

In analyses based on the superimposed vibrations, the damping matrix $C$ is not explicitly calculated, but the damping is directly determined in terms of the damping coefficient $\xi$, which is the ratio between actual and critical damping. Thus, the damping coefficient is a combination of the Rayleigh coefficients $\alpha$ and $\beta$, see Eq. (1), which depend on the basic damping coefficient and the natural frequencies of the different frequency modes. The coefficient $\alpha$, Eq. (2), represents the time delay and the coefficient $\beta$, Eq. (3), represents the initial value of the exponentially decaying sinusoidal oscillations [15]. To calculate the Rayleigh coefficients, a circular natural frequency is required, which can be calculated using Eq. (4) [16]. Since we also defined the damping coefficient $\tan \delta$ using the vibroacoustic analyses mentioned earlier [5], we calculated the basic damping coefficient $\zeta$ using Eq. (5).

$$
\begin{gathered}
\alpha=2 \xi \frac{\omega_{\max } \omega_{\min }}{\omega_{\max }+\omega_{\min }} \\
\beta=\frac{2 \xi}{\omega_{\max }+\omega_{\min }} \\
\omega_{i}=2 \pi f_{i}
\end{gathered}
$$

$\omega_{i}-$ circular natural frequency of the $i^{\text {th }}$ oscillation mode with frequency $f_{i}$

$$
\tan \delta=2 \xi
$$

According to preliminary vibroacoustic analyses [5], we decided to use the first and fourth modal frequencies in our analyses (Table II, Table III). 
Table I: Boundary conditions in ANSYS 2020 R2.

\begin{tabular}{|c|c|c|c|c|}
\hline String & Diameter $(\mathrm{mm})$ & Length $(\mathrm{mm})$ & String tension $(\mathrm{N})$ & String excitation force $(\mathrm{N})$ \\
\hline $\mathrm{G}$ & 0,4318 & 628 & 76,51 & 5 \\
\hline
\end{tabular}

Table II: Modal frequencies measured in the laboratory for each sample (Walnut / Ash).

\begin{tabular}{|c|c|c|}
\hline Mode & Walnut, $f_{i}(\mathrm{~Hz})$ & Ash, $f_{i}(\mathrm{~Hz})$ \\
\hline 1 & 748 & 923 \\
\hline 2 & 1754 & 1813 \\
\hline 3 & 2631 & 2672 \\
\hline 4 & 3675 & 3573 \\
\hline
\end{tabular}

Table III: Rayleigh coefficients $\alpha$ and $\beta$.

\begin{tabular}{|c|c|c|c|c|c|c|}
\hline \multirow{2}{*}{ Mode } & \multicolumn{3}{|c|}{ Walnut } & \multicolumn{3}{c|}{ Ash } \\
\cline { 2 - 6 } & $f_{1}$ & $\alpha$ & $\beta$ & $f_{1}$ & $\alpha$ & $\beta$ \\
\hline 1 & 748 & 26 & $1,17 \mathrm{E}-06$ & 923 & 23 & $6,90 \mathrm{E}-07$ \\
\hline
\end{tabular}

The numerical simulation was performed in four steps: setting up and conditioning the system; tensioning the string to tone $\mathrm{G}$; plucking the string with a force of $5 \mathrm{~N}$; and damping the string vibration until it "stopped". The maximum time interval of the last step was set to $1 \mathrm{~s}$ with a step size of $1 \mathrm{~ms}$.

The comparison of the decay of the vibration of the $G$ string as a function of the change of the body material (Fig. 8) confirms that the material of the guitar body affects the speed of the decay of the vibration of the string. Since we only changed the material properties and the damping factor for the guitar body in the numerical analyses, we can conclude that the model is built accordingly. We will show that in comparison with the measurement results this model is suitable for use in a guitar development system.

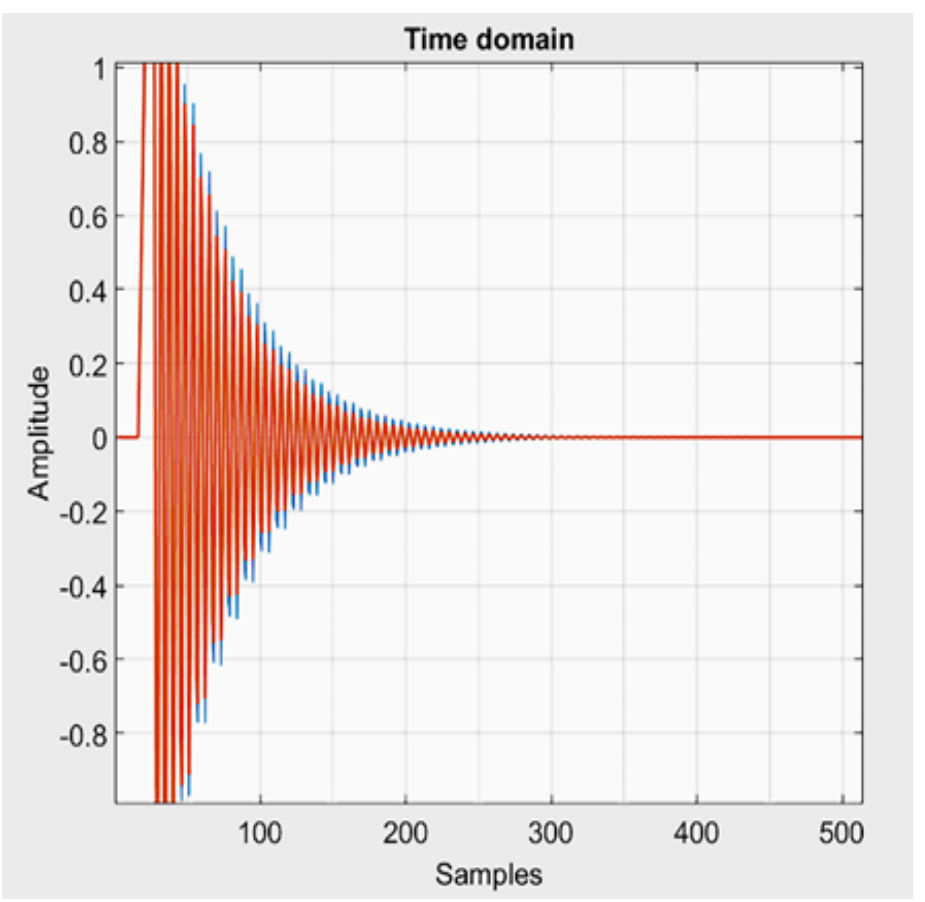

Figure 8: Comparison of the influence of the guitar body on the string $\mathrm{G}$ response (guitar body Ash and Walnut) in ANSYS 2020 R2. 


\section{VALIDATION OF NUMERICAL MODEL}

Based on the laboratory measurements carried out and the results of the numerical analyses, we can state that there are undoubtedly differences in the sound of guitars with the same body geometry but made of different materials with different mechanical and vibroacoustic properties, which are presented in Table IV.

Table IV: Relevant mechanical and vibroacoustic properties of the materials used.

\begin{tabular}{|c|c|c|}
\hline Property $\backslash$ Material & Walnut & Ash \\
\hline Density $\left(\mathrm{kg} / \mathrm{m}^{3}\right)$ & 623 & 807 \\
\hline$f_{1}\left(\mathrm{~s}^{-1}\right)$ & 748 & 923 \\
\hline $\tan \delta$ & 0.011 & 0.008 \\
\hline$E / \rho(\mathrm{GPa})$ & 9.79 & 14.93 \\
\hline
\end{tabular}

In order to facilitate the comparability of the values, we normalized them before comparison, thus preparing them for further processing [10].

First, if we compare the results of the laboratory measurements of the "body-string" configuration and the "body-neck-head-string" configuration, we see in Fig. 5 that the envelopes of the vibration decays are practically the same for the same pickup position. Therefore, the influence of the body vibrations on the change of the magnetic field of the pickup can be neglected when the pickup is placed in the guitar body.

The result of the measurements confirms the assumption [6] about the influence of the damping coefficient of the body material on the produced sound of the guitar. According to this, ash produces a brighter (bright) sound than walnut, which produces a warmer (warm) sound.
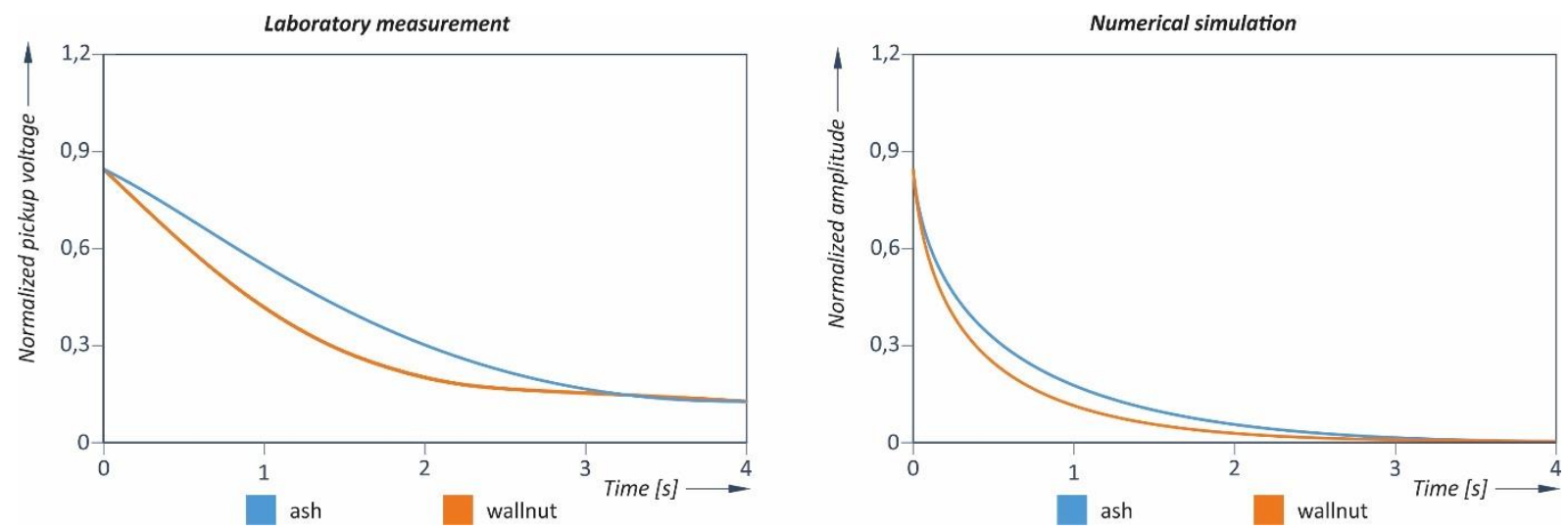

Figure 9: Comparison of oscillation decay envelopes for laboratory measurements and numerical simulation.

The same comparison can be made when analysing the numerical model data (Fig. 9). In this case, we compare the envelopes of the vibration decay under the same boundary conditions of the simulation with different material models. Also in this case, we can observe that the decay of the string vibration on the walnut body is faster, same as in the laboratory measurements.

Thus, the numerical model mimics the process of vibration decay as determined by laboratory measurements. Since it is designed to allow us to change the shape of the body and define the desired material models, it can be used in the design phase of the instrument, as envisaged in the design methodology presented in the introduction. 


\section{CONCLUSION}

The design and subsequent production of many musical instruments are still based primarily on the experience of master craftsmen, who often perform their work by feel. However, the development of modern materials, on the one hand, and advanced computer-aided technologies, on the other, provide designers with a wide range of possibilities for the purposeful design and development of various products, including musical instruments. Nowadays, the acoustic response of the instrument's components, and thus the characteristics of the expected sound, can be checked in a virtual environment at the design stage, taking into account all the design and aesthetic features of the instrument.

With the numerical model and protocol for electric guitar design presented in this paper, the designer has the possibility of a virtual analysis of the sound produced, which is not dependent on the subjective judgments of the listener, but is measurable. Using the results of laboratory measurements on sample instruments, the designer can assess whether the sound produced meets expectations and, if necessary, adjust the shape and material until a desired result in terms of sound image is achieved. As the work takes place in a virtual environment, this means that the development process is much faster and, above all, more predictable, as the instrument produced has a known sound.

There are several possible ways to continue the research presented in this paper. First, a current database of sound images of existing instruments could be expanded, which would make possible to adopt the same design methodology driven by acoustic measurements of the materials to be used on a wider range of instruments.

Another potential that should be further explored is to address the possibilities offered not only by different kinds of wood but also by a wide range of other (acoustic) materials that could be used for the body of an instrument, including (bio)composites and materials with unconventional Poisson's ratio.

Last but not least, we could reverse the whole process of instrument development and take as a starting point a specific sound or sound image that we want to achieve with a particular instrument. Then we would search in a virtual environment for the right combination of material and shape - in our case for the body of an electric guitar - to achieve the desired result. The matter, of course, is nowhere near as simple as it sounds. To realise this idea, we would need a sufficiently broad base of sound images, as well as different materials and their acoustic properties. Only then, with the help of some computer-aided optimization methods and probably artificial intelligence, we could search for the appropriate shape of the guitar body made of the chosen material, which of course should be conditioned by the main function and ergonomics (normal playing of the instrument is still possible) as well as by the aesthetic appearance. In any case, a great challenge for the future.

\section{ACKNOWLEDGEMENT}

The authors acknowledge the financial support from the Slovenian Research Agency (research core funding No. P2-0063).

\section{REFERENCES}

[1] Zorič, A. (2016). Process of electric guitar design, Master Thesis, University of Maribor, Faculty of Mechanical Engineering, Maribor (in Slovene)

[2] Noguchi, T.; Obataya, E.; Ando, K. (2012). Effects of aging on the vibrational properties of wood, Journal of Cultural Heritage, Vol. 13, No. 3 (Supplement), S21-S25, doi:10.1016/ j.culher.2012.02.008

[3] Bucur, V. (1996). Acoustics of wood, Materials Science Forum, Vols. 210-213, 101-108, doi:10.4028/www.scientific.net/MSF.210-213.101 
[4] Smardzewski, J.; Batko, W.; Kamisiński, T.; Flach, A.; Pilch, A.; Dziurka, D.; Mirski, R.; Roszyk, E.; Majewski, A. (2014). Experimental study of wood acoustic absorption characteristics, Holzforschung, Vol. 68, No. 4, 467-476, doi:10.1515/hf-2013-0160

[5] Zorič, A.; Kaljun, J.; Žveplan, E.; Straže, A. (2019). Selection of wood based on acoustic properties for the solid body of electric guitar, Archives of Acoustics, Vol. 44, No. 1, 51-58, doi:10.24425/aoa.2019.126351

[6] Zoric, A.; Kaljun, J. (2018). The influence of the acoustic properties of wood for the production of elecrtic solid body guitars, Katalinic, B. (Ed.), DAAAM International Scientific Book 2018, DAAAM International, Vienna, 195-210, doi:10.2507/daaam.scibook.2018.18

[7] Glamsch, J.; Deese, K.; Rieg, F. (2019). Methods for increased efficiency of FEM-based topology optimization, International Journal of Simulation Modelling, Vol. 18, No. 3, 453-463, doi:10.2507/IJSIMM18(3)482

[8] Kang, W. T.; Derani, M. N.; Ratnam, M. M. (2020). Effect of vibration on surface roughness in finish turning: simulation study, International Journal of Simulation Modelling, Vol. 19, No. 4, 595-606, doi:10.2507/IJSIMM19-4-531

[9] Rašović, N. (2021). Recommended layer thickness to the powder-based additive manufacturing using multi-attribute decision support, International Journal of Computer Integrated Manufacturing, Vol. 34, No. 5, 455-469, doi:10.1080/0951192X.2021.1891574

[10] Lemarquand, G.; Lemarquand, V. (2007). Calculation method of permanent-magnet pickups for electric guitars, IEEE Transactions on Magnetics, Vol. 43, No. 9, 3573-3578, doi:10.1109/ TMAG.2007.891671

[11] Harih, G.; Tada, M. (2015). Finite element evaluation of the effect of fingertip geometry on contact pressure during flat contact, International Journal for Numerical Methods in Biomedical Engineering, Vol. 31, No. 6, Paper e02712, doi:10.1002/CNM.2712

[12] Plesec, V.; Harih, G. (2020). Digital human models of lower limb amputees for socket modelling and simulations: an overview of current technologies, limitations and future possibilities, Advances in Transdisciplinary Engineering, Vol. 11, 348-358, doi:10.3233/ATDE200042

[13] Lerher, T.; Borovinsek, M.; Ficko, M.; Palcic, I. (2017). Parametric study of throughput performance in SBS/RS based on simulation, International Journal of Simulation Modelling, Vol. 16, No. 1, 96-107, doi:10.2507/IJSIMM16(1)8.372

[14] Otrin, M.; Boltežar, M. (2007). The vibration over a spatially curved steel wire with an outer band, Strojniski vestnik - Journal of Mechanical Engineering, Vol. 53, No. 10, 635-656

[15] Gibson, J. J. (1968). The Senses Considered as Perceptual Systems, George Allen \& Unwin, London

[16] Brancheriau, L.; Baillères, H.; Détienne, P.; Gril, J.; Kronland, R. (2006). Key signal and wood anatomy parameters related to the acoustic quality of wood for xylophone-type percussion instruments, Journal of Wood Science, Vol. 52, No. 3, 270-273, doi:10.1007/s10086-005-0755-2 